\title{
Appearance of enhanced Weiss oscillations in graphene: Theory
}

\author{
A. Matulis ${ }^{1,2, *}$ and F. M. Peeters ${ }^{1, \dagger}$ \\ ${ }^{1}$ Departement Fysica, Universiteit Antwerpen, Groenenborgerlaan 171, B-2020 Antwerpen, Belgium \\ ${ }^{2}$ Semiconductor Physics Institute, Goštauto 11, LT-01108 Vilnius, Lithuania
}

(Received 30 November 2006; revised manuscript received 15 February 2007; published 27 March 2007)

\begin{abstract}
The magnetoconductivity of a single graphene layer weakly modulated by a one-dimensional periodic potential is calculated. Pronounced Weiss oscillations periodic in the inverse magnetic field appear that are less damped by temperature as compared with the same oscillations found in a typical two-dimensional electron system with a standard parabolic energy spectrum.
\end{abstract}

DOI: 10.1103/PhysRevB.75.125429 PACS number(s): 72.20.My, 72.80.Rj, 73.50.Dn, 73.40.-c

\section{INTRODUCTION}

Recently the successful preparation of monolayer graphene films ${ }^{1-3}$ has generated a lot of activity in the physics of two-dimensional (2D) Dirac fermions. The massless energy spectrum and the specific density of states of electrons and holes described by the Dirac Hamiltonian enabled to study experimentally chiral tunneling and the Klein paradox in graphene, ${ }^{4}$ and led to the discovery of the unconventional "half-integer quantum Hall effect." ${ }^{\text {, }, 5,6}$ The presence of holes in graphene with a 2D Dirac-like spectrum was confirmed by measurements of de Haas-van Alphen and Shubnikov-de Haas (SdH) oscillations. ${ }^{7}$ These magnetic oscillations appear due to the interplay of the quantum Landau levels with the Fermi energy in the metal, and serve as a powerful technique to investigate the Fermi surface and the spectrum of electron excitations.

Another technique which was successfully used to obtain information on the electron spectrum of 2D systems is based on the interaction of electrons with artificially created periodic potentials with periods in the submicrometer range. Such electrical modulation of the 2D system was created by two interfering laser beams, ${ }^{8}$ or by depositing an array of parallel metallic strips on the surface, ${ }^{9}$ and led to the discovery of Weiss oscillations in the magnetoresistance. These oscillations are a consequence of the commensurability of the electron cyclotron orbit diameter at the Fermi energy and the period of the above electrical modulation. They were found to be periodic in the inverse magnetic field like the $\mathrm{SdH}$ oscillations, but have a different period versus electron density dependence. The period for Weiss oscillations varies with electron density $\left(n_{e}\right)$ as $\sqrt{n_{e}}$, whereas that of the $\mathrm{SdH}$ ones as $n_{e}$. Theoretical calculations of these oscillations were presented in Refs. 9-11, and it was shown that Weiss oscillations in the magnetoresistance for motion perpendicular to the oscillating potential can be understood as being a semiclassical effect. ${ }^{12,13}$

The Klein paradox in graphene where Dirac electrons can penetrate through potential barriers with a rather high probability implies that electrical control of Dirac electrons cannot be realized. Therefore, it is interesting to investigate the sensitivity of Dirac electrons on the electrical modulation of the graphene layer. Thus, we subjected the system to a periodic potential that introduces a new length scale and a new energy scale into the problem. We found that such a periodic potential also leads to Weiss oscillations in graphene which are even more pronounced than in typical 2D electron gases with a parabolic electron spectrum.

The paper is organized as follows. In Sec. II all necessary expressions for the magnetoconductivity calculation are given, and in Sec. III the obtained results for a graphene layer are compared with results for the standard 2D electron system. In Sec. IV the asymptotic expression valid in the quasiclassical limit is obtained. Simple classical explanations for the obtained results are presented in Sec. V. Our conclusions are presented in Sec. VI.

\section{ELECTRICAL MAGNETOTRANSPORT}

We consider the graphene layer within the single electron approximation where the low energy excitations are described by the two-dimensional (2D) Dirac-like Hamiltonian $^{4}$

$$
H_{0}=v_{F} \boldsymbol{\sigma} \cdot\left(-i \hbar \nabla+\frac{e}{c} \mathbf{A}\right)
$$

Here $\boldsymbol{\sigma}=\left\{\sigma_{x}, \sigma_{y}\right\}$ are the Pauli matrices, and the vector potential $\mathbf{A}=\{0, B x\}$ describing the magnetic field $\mathbf{B}=\{0,0, B\}$ perpendicular to the graphene layer is chosen in the Landau gauge. The parameter $v_{F}$ characterizes the electron velocity which is about 300 times smaller than the velocity of light. The total Hamiltonian consists of two parts

$$
H=H_{0}+U(x),
$$

where the additional potential

$$
U(x)=V_{0} \cos \left(2 \pi x / a_{0}\right)
$$

describes the static electrical modulation of our 2D system in the $x$ direction.

We consider this modulation as a small perturbation to the electron spectrum and calculate the conductivity correction caused by it. We use the Kubo formula ${ }^{14}$ to calculate the linear response to an applied external electric field. When the magnetic field is sufficiently strong that we have welldefined cyclotron orbits this formula can be simplified and related to the scattering induced migration of the Larmor circle center. It was shown in Ref. 13 that this so-called diffusive conductance gives the main contribution to the Weiss oscillations. Therefore, we shall restrict our calcula- 
tion to this diffusive conductivity and calculate the diagonal component by the following expression which is valid in the case of quasielastic scattering of electrons (see Ref. 15 for details):

$$
\sigma_{y y}=\frac{\beta e^{2}}{L_{x} L_{y}} \sum_{\zeta} f\left(E_{\zeta}\right)\left[1-f\left(E_{\zeta}\right)\right] \tau\left(E_{\zeta}\right)\left(v_{y}^{\zeta}\right)^{2} .
$$

$L_{x}, L_{y}$ are the dimensions of the layer, $\beta=\left(k_{B} T\right)^{-1}$ is the inverse temperature ( $k_{B}$ is the Boltzmann constant), $f(E)$ is the Fermi-Dirac distribution function, and $\tau(E)$ is the electron relaxation time. This diagonal component of the conductivity is caused by the influence of the electric modulation on the electron drift in crossed electric and magnetic fields and is an order of magnitude larger than the other diagonal component $\sigma_{x x}$ which appears due to the scattering on imperfections. Symbol $\zeta$ denotes the quantum numbers of the electron eigenstate, and diagonal matrix elements of the velocity operator $v_{y}(\zeta)$ can be calculated as derivatives of the energy eigenvalue over the corresponding electron momenta.

The Weiss oscillations can be seen in the diagonal component of the magnetoresistance which actually is the inverse conductivity tensor $\rho_{x x}=\sigma_{y y} /\left(\sigma_{x x} \sigma_{y y}-\sigma_{x y} \sigma_{y x}\right)$. In our case of small linear response it can be presented as $\rho_{x x}=\sigma_{y y} / \sigma_{x y}^{2}$, as the leading term of the Hall conductivity $\sigma_{x y} \sim 1 / B$ does not depend on the small electrical modulation.

The energy eigenvalues are defined through the solution of the stationary Schrödinger equation

$$
\{H-E\} \Psi(\mathbf{r})=0
$$

with total Hamiltonian (2). Using the perturbation expansion in $V_{0}$ powers we shall follow Ref. 13 and restrict ourselves by the lowest nonvanishing term contributing to $\sigma_{y y}$. For this purpose we must solve the zeroth-order Schrödinger equation with the Hamiltonian $H_{0}$. Due to the system homogeneity along the $y$ axis we substitute the eigenfunction as

$$
\Psi(\mathbf{r})=\frac{1}{\sqrt{L_{y}}} e^{i k_{y} y}\left(\begin{array}{l}
a(x) \\
b(x)
\end{array}\right),
$$

and transform the zero order eigenvalue problem into the following set of two ordinary differential equations for the wave function components:

$$
\begin{aligned}
& -i \hbar v_{F}\left(\frac{\partial}{\partial x}-i \frac{\partial}{\partial y}+\frac{x}{l^{2}}\right) b-E a=0, \\
& -i \hbar v_{F}\left(\frac{\partial}{\partial x}+i \frac{\partial}{\partial y}-\frac{x}{l^{2}}\right) a-E b=0,
\end{aligned}
$$

where $l=\sqrt{c \hbar / e B}$ is the magnetic length. Solution of these equations can be easily obtained making use of the analogy with the harmonic oscillator eigenvalue problem. It reads

$$
E_{n}^{D}=\frac{v_{F} \hbar}{l} \sqrt{2 n},
$$

$$
\Psi_{n, k_{y}}(\mathbf{r})=\frac{e^{i k_{y} y}}{\sqrt{2 L_{y} l}}\left(\begin{array}{c}
-i \Phi_{n-1}\left[\left(x+x_{0}\right) / l\right] \\
\Phi_{n}\left[\left(x+x_{0}\right) / l\right]
\end{array}\right)
$$

where

$$
\Phi_{n}(x)=\frac{e^{-x^{2} / 2}}{\sqrt{2^{n} n ! \sqrt{\pi}}} H_{n}(x)
$$

is expressed in the normalized Hermitian polynomials, and $x_{0}=l^{2} k_{y}$ indicates the localization of the electron in the $x$ direction.

In first order perturbation expansion one must add to the above energy eigenvalue the diagonal matrix element of the potential (3) calculated with the above eigenfunctions,

$$
\begin{aligned}
\Delta E_{n k_{y}} & =\int_{-\infty}^{\infty} d x \int_{0}^{L_{y}} d y \Psi_{n, k_{y}}^{+}(\mathbf{r}) U(x) \Psi_{n, k_{y}}(\mathbf{r}) \\
& =\frac{V_{0}}{2} \cos \left(K x_{0}\right) e^{-u / 2}\left[L_{n}(u)+L_{n-1}(u)\right],
\end{aligned}
$$

where $K=2 \pi / a_{0}, u=K^{2} l^{2} / 2$, and $L_{n}(u)$ is a Laguerre polynomial. This energy correction makes the degenerate Landau levels $k_{y}$ dependent, expands them into bands, what finally leads to nonzero velocities,

$$
\begin{aligned}
v_{y}^{\zeta} \equiv v_{y}^{n, k_{y}} & =\frac{1}{\hbar} \frac{\partial}{\partial k_{y}} \Delta E_{n, k_{y}} \\
& =-\frac{V_{0}}{\hbar K} u e^{-u / 2}\left[L_{n}(u)+L_{n-1}(u)\right] \sin \left(K x_{0}\right) .
\end{aligned}
$$

Now substituting the velocities in Eq. (4) and specifying the summation over quantum numbers as

$$
\sum_{\zeta}=\frac{L_{y}}{2 \pi} \int_{0}^{L_{x} / l^{2}} d k_{y} \sum_{n=0}^{\infty}
$$

we obtain the final expression for the diffusion contribution to the dc conductivity,

$$
\sigma_{y y}=A_{0} \Phi,
$$

where

$$
A_{0}=8 \pi^{2} \frac{e^{2}}{h} \frac{V_{0}^{2} \tau \beta}{\hbar}
$$

and the function

$$
\Phi=\frac{1}{4} u e^{-u} \sum_{n=0}^{\infty} \frac{g\left(E_{n}\right)}{\left[g\left(E_{n}\right)+1\right]^{2}}\left[L_{n}(u)+L_{n-1}(u)\right]^{2}
$$

will be considered as a dimensionless conductivity. In this expression we introduced the following exponential function $g(E)=\exp \left[\beta\left(E-E_{F}\right)\right]$ where $E_{F}$ is the Fermi energy.

\section{COMPARISON WITH THE USUAL WEISS OSCILLATIONS}

It is interesting to compare the obtained expression for $\mathrm{dc}$ conductivity with the same conductivity calculated in Ref. 13 


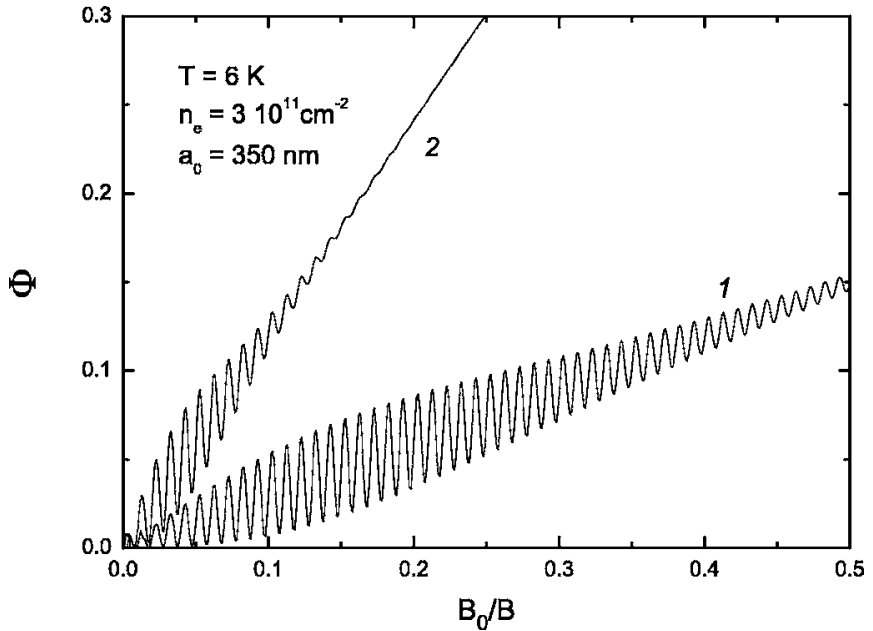

FIG. 1. The dimensionless conductivity versus inverse magnetic field: curve 1, Dirac electrons; curve 2, electrons with parabolic energy spectrum.

for the case of a 2D electron system localized at the interface between two semiconductors and having a parabolic energy dependence. Comparing velocity expression (11) with the equivalent one in Ref. 13 [see Eq. (4)] we notice that the diffusive conductivity for the system of Dirac electrons differs from the one for the standard interface case by replacement of the Laguerre polynomial $L_{n}(u)$ by the average of two successive polynomials $\left[L_{n}(u)+L_{n-1}(u)\right] / 2$. And of course, two different expressions for the Landau level energies [Eq. (8a) for Dirac electrons and $E_{n}^{P}=\hbar \omega_{c}(n+1 / 2)$ with $\omega_{c}$ $=e B / m c$ for the parabolic electron spectrum case] must be used.

These differences in the expressions lead to essentially different results for the dimensionless conductivity as shown in Fig. 1. The results are shown as a function of the inverse magnetic field for the temperature $T=6 \mathrm{~K}$, electron density $n_{e}=3 \times 10^{11} \mathrm{~cm}^{-2}$, and the period of electric modulation $a_{0}$ $=350 \mathrm{~nm}$. The dimensionless magnetic field $b=B / B_{0}$ is introduced using the characteristic magnetic field $B_{0}=c \hbar / e a_{0}^{2}$ corresponding to the magnetic length equal to the modulation period $a_{0}$, which for the above case is equal to $B_{0}$ $=0.0054 \mathrm{~T}$.

We see that in graphene (curve 1) the Weiss oscillations are more pronounced as compared with the system of electrons with the standard parabolic energy spectrum (curve 2). Furthermore, the Weiss oscillations in graphene are much more robust with respect to temperature damping in the quasiclassical region of small magnetic fields. The physical reasons for these differences are in different Fermi velocities of Dirac and standard electrons. In order to confirm the above statement we shall consider the asymptotic behavior of the Weiss oscillations in the quasiclassical region which according to Refs. 9, 12, and 13 describes the main features of the above oscillations and which also allow for explicit analytical expressions.

\section{ASYMPTOTIC EXPRESSIONS}

The asymptotic expression for the conductivity (15) is obtained following the approach of Ref. 13 for the case of standard electrons. That approach is applicable when many Landau levels are filled, and it is based on the following asymptotic expression for the Laguerre polynomials:

$$
e^{-u / 2} L_{n}(u) \rightarrow \frac{1}{\sqrt{\pi \sqrt{n u}}} \cos (2 \sqrt{n u}-\pi / 4)
$$

Taking the continuum limit

$$
n \rightarrow \frac{1}{2}\left(\frac{l E}{v_{F} \hbar}\right)^{2}, \quad \sum_{n=0}^{\infty} \rightarrow\left(\frac{l}{v_{F} \hbar}\right)^{2} \int_{0}^{\infty} E d E
$$

and keeping in mind that $u=2 \pi^{2} / b$, we transform Eq. (15) into the following integral:

$$
\begin{aligned}
\Phi= & \frac{\sqrt{u}}{\pi}\left(\frac{l}{v_{F} \hbar}\right)^{2} \int_{0}^{\infty} \frac{g(E) E d E}{[g(E)+1]^{2} \sqrt{n}} \\
& \times \cos ^{2}\left(\frac{1}{2} \sqrt{\frac{u}{n}}\right) \cos ^{2}\left(2 \sqrt{n u}-\frac{\pi}{4}\right) \\
= & \frac{2 a_{0}}{v_{F} \hbar b} \int_{0}^{\infty} \frac{g(E) d E}{[g(E)+1]^{2}} \cos ^{2}\left(\frac{\pi v_{F} \hbar}{E a_{0}}\right) \\
& \times \cos ^{2}\left(\frac{2 \pi a_{0} E}{v_{F} b \hbar}-\frac{\pi}{4}\right) .
\end{aligned}
$$

Now assuming that the temperature is low $\left(\beta^{-1} \ll E_{F}\right)$ and replacing $E=E_{F}+s \beta^{-1}$ we rewrite the above integral as

$$
\Phi=\frac{2 a_{0}}{v_{F} \hbar b \beta} \cos ^{2}\left(\frac{\pi}{p}\right) \int_{-\infty}^{\infty} \frac{d s e^{s}}{\left(e^{s}+1\right)^{2}} \cos ^{2}\left(\frac{2 \pi p}{b}-\frac{\pi}{4}+\frac{2 \pi p}{b \beta} s\right),
$$

where

$$
p=\frac{E_{F} a_{0}}{v_{F} \hbar}=k_{F} a_{0}=\sqrt{2 \pi n_{e}} a_{0}
$$

stands for the dimensionless Fermi momentum of the electron. Note in Eq. (19) we replaced all energies $E$ by the Fermi energy $E_{F}$ except that one which is included in the last cosine function, where the small energy correction can influence the damping of the Weiss oscillations.

The obtained expression for the dimensionless conductivity can be calculated using the standard integral

$$
\int_{0}^{\infty} \frac{\cos (a x)}{\cosh (\beta x)} d x=\frac{\pi}{2 \beta \sinh (a \pi / 2 \beta)},
$$

which leads to

$$
\begin{aligned}
\Phi= & \frac{T}{4 \pi^{2} T_{D}} \cos ^{2}\left(\frac{\pi}{p}\right)\left\{\left[1-A\left(\frac{T}{T_{D}}\right)\right]\right. \\
& \left.+2 A\left(\frac{T}{T_{D}}\right) \cos ^{2}\left[2 \pi\left(\frac{p}{b}-\frac{1}{8}\right)\right]\right\},
\end{aligned}
$$

where 


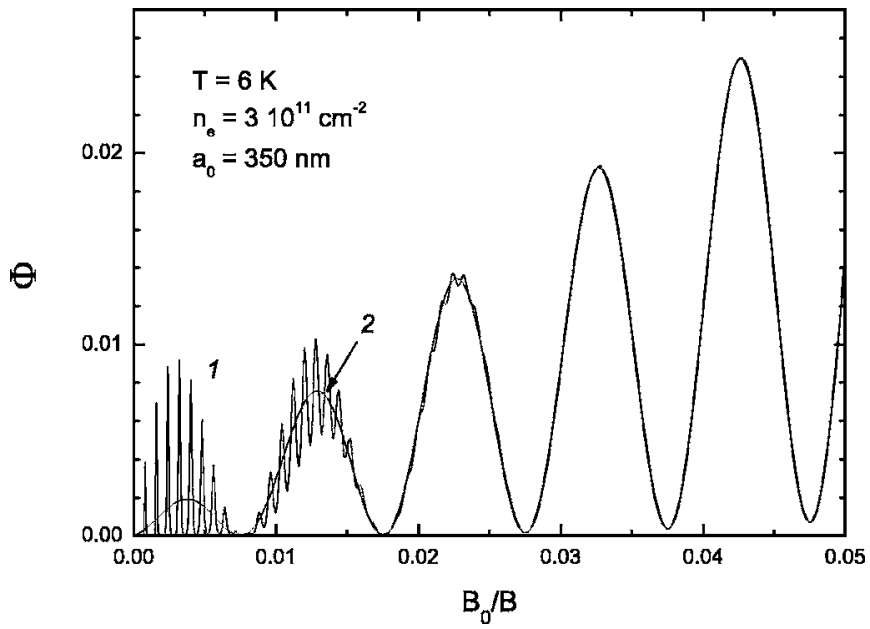

FIG. 2. The graphene conductivity as a function of the inverse magnetic field: curve 1, exact solution (15); curve 2, asymptotic expression (22).

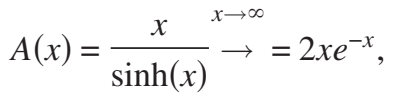

and the symbol $T_{D}$ defined as

$$
k_{B} T_{D}=\frac{b \hbar}{4 \pi^{2} a_{0}} v_{F}
$$

gives the temperature scale for damping of the Weiss oscillations.

The validity of the asymptotic expression is seen in Fig. 2 where the Weiss oscillations for the Dirac electrons (curve 1) are shown together with their asymptotic expression (curve 2) for the same parameter values as in Fig. 1 in the region of strong magnetic fields where deviations of the asymptotic expression to the exact expression are largest.

We see that the coincidence of the exact result and its asymptotic expression is rather good everywhere except the region of very strong magnetic field where $\mathrm{SdH}$ oscillations become superimposed on top of the Weiss oscillations.

Here, we calculated only the extra contribution to the conductivity resulting from the electrical one-dimensional modulation and as such the pure $\mathrm{SdH}$ oscillations are not included in our results. Nevertheless, this extra contribution to the conductivity can exhibit $\mathrm{SdH}$-type oscillations and it is interesting to highlight their character. For this purpose in Fig. 3 we show these oscillations for Dirac electrons (solid curve) and standard ones (dashed curve) in more detail in the region of strong magnetic field.

We see a clear $\pi$ phase shift between these two oscillations in agreement with the known behavior of $\mathrm{SdH}$ oscillations ${ }^{5}$ caused by the presence of half-filled zero energy Landau level for Dirac electrons.

We compare now the obtained result for the conductivity of the Dirac electron system with similar asymptotic result for the system of electrons with a standard parabolic energy spectrum taken from Ref. 13 which can be presented as follows:

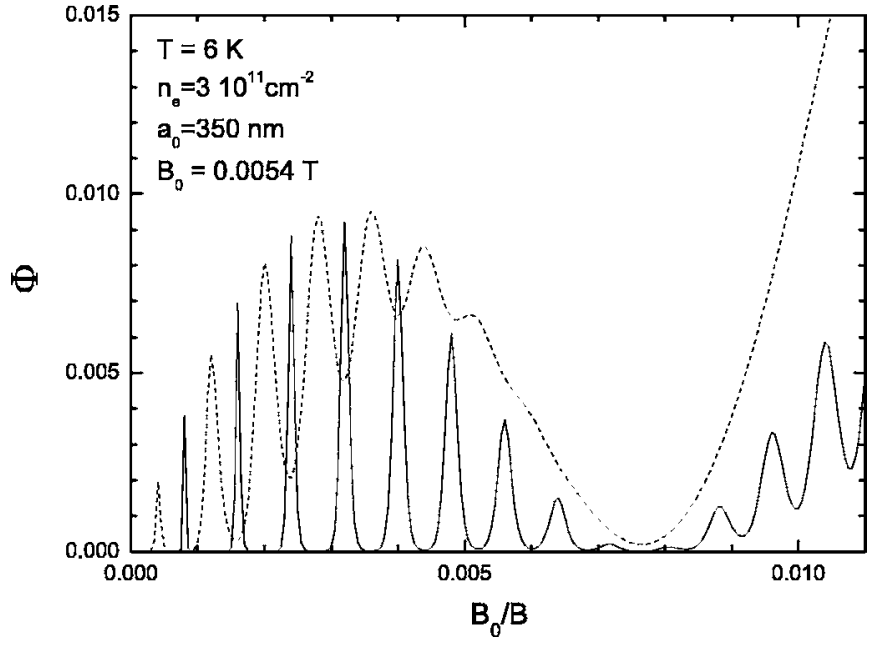

FIG. 3. The conductivity $\sigma_{x x}$ as a function of the inverse magnetic field in the region of very strong magnetic field: solid curve, Dirac electrons; dashed curve, standard electrons.

$$
\Phi=\frac{T}{4 \pi^{2} T_{P}}\left\{\left[1-A\left(\frac{T}{T_{P}}\right)\right]+2 A\left(\frac{T}{T_{P}}\right) \cos ^{2}\left[2 \pi\left(\frac{p}{b}-\frac{1}{8}\right)\right]\right\},
$$

where the critical temperature reads

$$
k_{B} T_{P}=\frac{b p \hbar^{2}}{4 \pi^{2} m a_{0}^{2}} .
$$

Keeping in mind that

$$
\frac{p}{m}=\frac{k_{F} a_{0}}{m}=\frac{a_{0}}{\hbar} v_{F}^{P}
$$

where $v_{F}^{P}$ is the velocity of the electrons on the Fermi surface for a parabolic energy system, the critical temperature can be presented as

$$
k_{B} T_{P}=\frac{b \hbar}{4 \pi^{2} a_{0}} v_{F}^{P} .
$$

For parameters used in Fig. 1 we have $k_{F} \sim 1.4 \times 10^{6}, p$ $\sim 50$, and $\cos (\pi / p) \sim 1$. Thus the asymptotic behavior of the dimensionless conductivity for Dirac electrons (22) and standard electrons with parabolic energy spectrum (25) is characterized by different temperature scales.

We see from Eqs. (22) and (25) that the period and the phase of Weiss oscillations are the same for both the Dirac and the standard electron cases that are also evident from Figs. 1 and 3. The period can be easily estimated equating the increment of the cosine argument to $\pi$,

$$
2 \pi \Delta \frac{p}{b}=\pi
$$

and taking into account the definitions of the dimensionless Fermi momentum (20) and magnetic field $b$ immediately leads to 


$$
\Delta \frac{\sqrt{n_{e}}}{B}=\frac{\sqrt{2 / \pi}}{B_{0} a_{0}}=\sqrt{\frac{2}{\pi}} \frac{e a_{0}}{c \hbar} .
$$

This coincidence of the periods confirms the quasiclassical nature of Weiss oscillations and aggravates the discrimination between Dirac and standard electrons.

Nevertheless, there is a distinct feature in the Weiss oscillations which enables to discriminate Dirac electrons from the usual electrons. Comparing Eqs. (24) and (28) we see that

$$
\frac{T_{P}}{T_{D}}=\frac{v_{F}^{P}}{v_{F}}
$$

or the ratio of critical temperatures for the standard and Dirac electrons is equal to the ratio of the corresponding velocities at the Fermi surface. This can be estimated as

$$
\frac{T_{P}}{T_{D}}=\frac{\hbar \sqrt{2 \pi n_{e}}}{v_{F} m a_{0}}
$$

and for typical cases it is less than unity. For instance, in the case of the parameters used in Fig. 1 it is $T_{P} / T_{D} \sim 0.24$ that explains the different slope and damping of the Weiss oscillations.

\section{QUASICLASSICAL EXPLANATION}

The obtained results can be understood from a simple physical picture. In order to estimate the oscillation period we write down the momentum of the electrons on the Fermi surface,

$$
p_{F}=m \omega_{c} R_{c},
$$

which is identical in both cases. Then it follows that the radius of the electron orbit is

$$
R_{c}=\frac{p_{F}}{m \omega_{c}}=\frac{\hbar k_{F} c}{e B}=\frac{p l^{2}}{a_{0}} .
$$

The physical reason for the appearance of the Weiss oscillations is the commensurability of the electron orbit diameter with the period of the electrical modulation, consequently, the argument of the cosine function must be proportional to

$$
\frac{R_{c}}{a_{0}}=p \frac{l^{2}}{a_{0}^{2}}=\frac{p}{b},
$$

which we can see in both expressions (22) and (25).

The damping factor of the oscillations can be estimated as follows. Due to thermal fluctuations there are electron orbits with various radii. The effective damping can be estimated as the ratio of the above broadening of the orbit to the period of the modulation,

$$
\delta=\frac{1}{a_{0}} \frac{\partial R_{c}}{\partial E_{F}} \beta^{-1}
$$
reads

In the case of standard electrons (when $E_{F}=p_{F}^{2} / 2 m$ ) it

$$
\delta_{P}=\frac{m}{a_{0} \beta p_{F}} \frac{\partial R_{c}}{\partial p_{F}}=\frac{m}{a_{0} \beta \hbar k_{F} m \omega_{c}}=\frac{k_{B} T}{p \hbar \omega_{c}}=\frac{m a_{0}^{2}}{\hbar^{2} p b} k_{B} T,
$$

in agreement with the definition of the critical temperature for standard electrons (26).

In the case of Dirac electrons (when $E_{F}=v_{F} p_{F}$ ) the above damping parameter can be estimated as

$$
\delta_{D}=\frac{1}{\beta a_{0} v_{F}} \frac{\partial R_{c}}{\partial p_{F}}=\frac{c k_{B} T}{a_{0} v_{F} e B}=\frac{a_{0}}{\hbar b v_{F}} k_{B} T,
$$

which coincides with the earlier obtained result for the critical temperature up to the same constant $4 \pi^{2}$. Now dividing Eq. (37) by Eq. (38) we obtain the same critical temperature ratio dependence on the ratio of the Fermi velocities (31) that confirms the quasiclassical nature of the Weiss oscillations.

\section{CONCLUSIONS}

In conclusion, we studied the Weiss oscillations in an electrically modulated single layer of graphene. It was shown that the static conductivity oscillations are periodic in $1 / B$ with period varying with electron density as $\sqrt{n_{e}}$ like in the 2D electron system with standard parabolic energy spectrum. Due to the larger Fermi velocity the conductivity oscillations of the Dirac electron system are more pronounced and less damped as compared with the 2D system of electrons with parabolic energy spectrum for analogous parameters. The found Dirac electron sensitivity to the electric perturbation does not contradict the Klein paradox, because in contrast to electron tunneling through barriers where both the electron and the hole nature of the excitation plays a role, only the electrons at the Fermi energy are responsible for the conductivity and for the studied Weiss oscillations. Thus, their behavior in the electric field is not overshadowed by the admixture of the hole states.

\section{ACKNOWLEDGMENTS}

Part of this work is supported by the Flemish Science Foundation (FWO-Vl) and the Belgian Science policy (IAP).

\footnotetext{
*Electronic address: amatulis@takas.lt

'Electronic address: francois.peeters@ua.ac.be

${ }^{1}$ K. S. Novoselov, A. K. Geim, S. V. Morozov, D. Jiang, Y. Zhang, S. V. Dubonos, I. V. Grigorieva, and A. A. Firsov, Science 306, 666 (2004).
}

${ }^{2}$ Y. Zhang, Y. W. Tan, H. Stormer, and P. Kim, Nature (London) 438, 201 (2005).

${ }^{3}$ C. Berger, Z. Song, X. Li, X. Wu, N. Brown, C. Naud, D. Mayou, T. Li, J. Hass, A. N. Marchenkov, E. H. Conrad, P. N. First, and W. A. de Heer, Science 312, 1191 (2006). 
${ }^{4}$ M. I. Katsnelson, K. S. Novoselov, and A. K. Geim, Nat. Phys. 2, 620 (2006); J. M. Pereira, Jr., V. Mlinar, F. M. Peeters, and P. Vasilopoulos, Phys. Rev. B 74, 045424 (2006).

${ }^{5}$ K. S. Novoselov, A. K. Geim, S. V. Morozov, D. Jiang, M. I. Katsnelson, I. V. Grigorieva, S. V. Dubonos, and A. A. Firsov, Nature (London) 438, 197 (2005).

${ }^{6}$ V. P. Gusynin and S. G. Sharapov, Phys. Rev. Lett. 95, 146801 (2005); N. M. R. Peres, F. Guinea, and A. H. Castro Neto, Phys. Rev. B 73, 125411 (2006).

${ }^{7}$ S. G. Sharapov, V. P. Gusynin, and H. Beck, Phys. Rev. B 69, 075104 (2004).

${ }^{8}$ D. Weiss, K. von Klitzing, K. Ploog, and G. Weinmann, Europhys. Lett. 8, 179 (1989); in High Magnetic Fields in Semicon- ductor Physics II, edited by G. Landwehr (Springer-Verlag, Wurtzburg, 1989), p. 357.

${ }^{9}$ R. W. Winkler, J. P. Kotthaus, and K. Ploog, Phys. Rev. Lett. 62, 1177 (1989).

${ }^{10}$ R. R. Gerhardts, D. Weiss, and K. v. Klitzing, Phys. Rev. Lett. 62, 1173 (1989).

${ }^{11}$ P. Vasilopoulos and F. M. Peeters, Phys. Rev. Lett. 63, 2120 (1989).

${ }^{12}$ C. W. J. Beenakker, Phys. Rev. Lett. 62, 2020 (1989).

${ }^{13}$ F. M. Peeters and P. Vasilopoulos, Phys. Rev. B 46, 4667 (1992).

${ }^{14}$ R. Kubo, H. Hasegawa, and N. Hashitsume, J. Phys. Soc. Jpn. 14, 56 (1959).

${ }^{15}$ M. Charbonneau, K. M. Van Vliet, and P. Vasilopoulos, J. Math. Phys. 23, 318 (1982). 